\title{
O Mini-CEX na avaliação de habilidades clínicas na graduação médica
}

\author{
Mini-CEX in the evaluation of clinical skills in medical graduation
}

\author{
Abdias Pereira Diniz Neto ${ }^{1}$, Clarice Freitas Timóteo de Lima ${ }^{1}$, Kelly Rayane Paiva Carvalho ${ }^{1}$, \\ Ruana Manso Porfírio dos Santos ${ }^{1}$, Roberto Santos Lima ${ }^{2}$, Raphaella Amanda Maria Leite \\ Fernandes ${ }^{2}$, Alexandre César Vieira de Sales ${ }^{3}$
}

Diniz Neto AP, Lima CFT, Carvalho KRP, Santos RMP, Lima RS, Fernandes RAML, Sales ACV. O Mini-CEX na avaliação de habilidades clínicas na graduação médica / Mini-CEX in the evaluation of clinical skills in medical graduation. Rev Med (São Paulo). 2020 jan.-fev.;99(1):35-9.

RESUMO: Introdução: Instrumentos de avaliação de habilidades clínicas na medicina são importantes para a análise do conhecimento, habilidades e atitudes do estudante. Dentre eles, o Mini Clinical Evaluation Exercise (mini-CEX) vem se destacando por avaliar o estudante em ambiente real de prática, além de fornecer feedback imediato. Objetivo: Realizar uma revisão sistemática da literatura sobre a aplicação do instrumento mini-CEX na graduação do curso de medicina. Métodos: As bases de dados pesquisadas foram: Eric, Pubmed e Science Direct. Através de duas estratégias de busca diferentes: Busca A, com período de pesquisa de 2011 a 2016 e a Busca B, com intervalo de 2006 a 2016, foram incluídos artigos que abordam o miniCEX como instrumento de avaliação das habilidades clínicas em acadêmicos de medicina. Foram consideradas as características gerais dos estudos, o uso do mini-CEX na graduação médica e os critérios avaliados por esse instrumento. Resultados: Dos 140 artigos encontrados, três atenderam aos critérios de inclusão. Os estudos avaliaram as habilidades clínicas dos estudantes de medicina com o uso do mini-CEX, a partir da: habilidade de comunicação, atitude/profissionalismo, humanização, raciocínio clínico, habilidades de aconselhamento, organização/eficiência e competência clínica geral. Dentre essas competências, a anamnese, o exame físico e a capacidade de comunicação são melhores avaliados pelo mini-CEX quando comparado a outros métodos de avaliação de competências. Além disso, competências como comportamentos pessoal e profissional obtiveram as maiores pontuações gerais. Conclusões. O mini-CEX parece ser uma ferramenta eficaz, de fácil aplicação e confiável na avaliação das habilidades clínicas dos acadêmicos de medicina.

Descritores: Educação de graduação em medicina; Estudos de avaliação; Exames médicos; Competência clínica/normas; Avaliação educacional; Estudantes de medicina.
ABSTRACT: Introduction. Assessment methods of clinical skills in medicine are important for the analysis of students' knowledge, skills and attitudes. Among these methods, the Mini Clinical Evaluation Exercise (mini-CEX) has been highlighted for evaluating the student in real practice environment, in addition to providing immediate feedback. Objective. To conduct a systematic review of the literature of the application of Mini Clinical Evaluation Exercise instrument in medical school graduation. Methods. Eric, Pubmed and Science Direct were searched. Through two differents search strategies: Search A, with publications from 2011 to 2016, and Search B, with publications from 2006 to 2016, including articles with mini$\mathrm{CEX}$ as an instrument for evaluating clinical skills in medical students. General characteristics of the studies, the use of the mini-CEX in medical graduation and the evaluated abilities were examined. Results. Of the 140 studies identified, 3 met the inclusion criteria. The studies evaluated clinical skills of medical students using mini-CEX, from the following: communication skills, personal and professional behavior, humanization, clinical judgement, counseling skills, organization and efficiency and overall clinical care. Among these skills, history taking, physical examination and the communication skills are best evaluated by mini-CEX when compared to other skills assessment methods. Finally, competences such as personal and professional behaviours obtained the highest overall scores. Conclusions. The mini-CEX is an effective tool, easy to apply and reliable on the evaluation of clinical skills of the medical academics.

Keywords: Education, medical, undergraduate; Evaluation studies; Clinical competence/standards; Medical examination; Educational measurement; Students, medical.

1. Acadêmico de Medicina da Universidade Federal de Pernambuco (UFPE) - Campus Acadêmico do Agreste (CAA) - Núcleo Ciências da Vida (NCV) - Caruaru, PE, BR. ORCID: Diniz Neto AP - https://orcid.org/0000-0002-3557-7964; Lima CFT - https://orcid.org/0000-0002-3891-6714; Carvalho KRP - https://orcid.org/0000-0001-5938-6524; Santos RMP - https://orcid.org/0000-0001-7812-4781. Email: abdiasdiniz@gmail.com, clariceft1@ hotmail.com, rayanecarvalho0301@gmail.com, ruanaporfirio@gmail.com.

2. Professor Auxiliar da Universidade Federal de Pernambuco (UFPE) - Campus Acadêmico do Agreste (CAA) - Núcleo Ciências da Vida (NCV). Caruaru, PE, BR. ORCID: Lima RS - https://orcid.org/0000-0001-7228-2682; Fernandes RAML - https://orcid.org/0000-0001-6306-0703. Email: robertosantoslima@gmail.com, raphaella.fernandes00@gmail.com.

3. Professor Assistente de Oncologia da Universidade Federal de Pernambuco (UFPE) - Campus Acadêmico do Agreste (CAA) - Núcleo Ciências da Vida (NCV). Caruaru, PE, BR. ORCID: https://orcid.org/0000-0002-3085-7967. Email: ac.sales@uol.com.br.

Endereço para correspondência: Abdias Pereira Diniz Neto. Rua Enedino de Freitas, 237. Centro, Pesqueira, PE, BR. CEP: 55200-000. Email: abdiasdiniz@gmail.com . 


\section{INTRODUÇÃO} American Board of Internal Medicine (ABIM),
em 1972, reviu o seu sistema de avaliação de residentes de medicina e aprovou o Clinical Exercise Evaluation (CEX), o qual consistia numa avaliação do exame clínico realizado pelo aluno no leito do paciente, a fim de medir as competências clínicas do residente ${ }^{1}$. No entanto, após seu uso, esse método foi considerado inadequado, em parte, pois além de demandar cerca de duas horas para ser concluído, os resultados apresentavam divergências entre os avaliadores, apesar de os mesmos usarem o mesmo instrumento ${ }^{2}$.

$\mathrm{Na}$ década de 90, também pela ABIM, foi desenvolvido um método considerado uma variação do tradicional CEX: o Mini Clinical Evaluation Exercise (mini-CEX). Esse instrumento é de observação direta de desempenho, no qual permite a avaliação do estudante pelo professor, enquanto aquele realiza uma consulta objetiva e rápida, focada em determinada necessidade do paciente. Essa ferramenta tornou-se importante pois tenta reproduzir, da maneira mais fiel possível, a rotina do acadêmico em seu futuro local de trabalho ${ }^{1,2,3}$. O miniCEX avalia sete competências: realização da anamnese, habilidade de comunicação, habilidade ao exame físico, raciocínio clínico, conduta ética, organização/eficiência e competência clínica geral. Na sequência da observação da consulta, o professor/observador pontua cada um desses diferentes domínios e transmite, de imediato, um feedback ao estudante sobre seu desempenho, sugerindo melhoras ${ }^{2}$.

Esse método de avaliação já está consolidado para o uso com residentes do curso médico, e vem ganhando espaço com seu uso durante a graduação de medicina, sobretudo por servir não apenas como um instrumento avaliativo, mas também formativo na graduação devido à possibilidade de ofertar um feedback ao estudante, sugerindo melhoras em alguma das sete competências avaliadas ${ }^{3}$.

Devido à escassez de trabalhos que relacionam a aplicabilidade do mini-CEX na graduação médica, esse estudo tem como objetivo realizar uma revisão sistemática da literatura sobre o uso desse instrumento nos cursos de medicina, com a finalidade de evidenciar os pontos importantes que podem subsidiar sua aplicação.

\section{METODOLOGIA}

Trata-se de um estudo de revisão sistemática, segundo a metodologia proposta pelo Instituto Cochrane ${ }^{4}$. Para identificar os artigos acerca do tema, foram realizadas buscas nas seguintes bases de dados: Eric, Pubmed e Science Direct, em janeiro de 2017, com duas estratégias de busca. Na primeira estratégia (busca A), utilizaram-se os seguintes descritores: "mini-Cex and clinical skills" e, na segunda (busca B), "mini-Cex + clinical skills + assessment methods + medical education + undergraduate". Apenas termos em inglês foram utilizados. Não foram realizadas buscas manuais nas referências bibliográficas dos artigos encontrados.

Foram incluídos artigos que trazem o mini-CEX como instrumento de avaliação das habilidades clínicas em acadêmicos de medicina, com metodologia claramente descrita, publicados em periódicos na língua inglesa e espanhola, no período de 5 anos (na busca A) e 10 anos (na busca B). Para exclusão dos artigos, foram empregados os seguintes critérios: relatos de caso, outras revisões sistemáticas ou metanálises; estudos multiprofissionais ou que tratassem de outras áreas da saúde que não a médica; estudos que abrangessem a formação de examinadores, bem como artigos que discutissem os pontos de vista dos examinadores e/ou alunos sobre o tema, ou fizessem apenas comparação do mini-CEX com outros métodos avaliativos.

Após essa consulta às bases de dados, utilizando as estratégias de busca e critérios de inclusão e exclusão descritos, foram identificados estudos que apresentavam duplicidade entre as bases. Todos os resumos resultantes foram lidos e, nos casos em que só essa leitura não foi suficiente para definir se o artigo deveria ser incluído segundo os critérios supracitados, foi realizada a leitura na íntegra do artigo a fim de estabelecer sua elegibilidade. Nos casos em que o resumo era suficiente, os artigos eram selecionados e era obtida sua versão integral para confirmação da elegibilidade. Em casos de discordância entre os pesquisadores, a tentativa de consenso foi preferida. Não sendo possível, a opinião da maioria dos autores do presente estudo prevaleceu.

\section{RESULTADOS}

Após a realização da primeira busca (Busca A), tendo como descritor "mini-CEX and clinical skills", identificaram-se 72 artigos, dos quais quatro foram elegíveis para estudo e 68 foram excluídos por preencherem critérios de exclusão ou não se referirem ao tema: dois artigos por tratarem de planejamento de cuidados no mini-CEX, dois artigos por centrarem seus resultados na formação dos avaliadores, 17 artigos por analisarem o ponto de vista do avaliador no uso do instrumento, 26 artigos por tratarem do uso do mini-CEX para residentes, um por abordar o aspecto cultural, cinco artigos por tratarem de áreas não médicas, 10 artigos por investigarem majoritariamente a visão do estudante, dois por serem metanálise, um por refletir a assistência ao paciente, um por ser revisão da literatura e um por ser revisão sistemática. Selecionados os quatro artigos elegíveis dessa primeira busca, foi feita a leitura na íntegra, tendo por fim três exclusões por não se enquadrarem nos critérios de elegibilidade citados na metodologia, tendo, portanto, apenas um estudo válido para essa revisão. Já na segunda busca (Busca B), feita com os seguintes descritores: "mini-Cex + clinical skills + assessment methods + medical 
education + undergraduate", foram encontrados 68 artigos, dentre os quais 12 foram selecionados para leitura integral de seus conteúdos, e 56 foram excluídos por preencherem critérios de exclusão ou não se referirem ao tema: um por ter centrado na formação de educadores, seis por refletirem o ponto de vista do avaliador no uso do mini-CEX, 24 por tratarem sobre o uso o instrumento na residência médica, 16 por abordarem o uso do mini-CEX em outra área da saúde, sete por focarem sua análise em outros instrumentos comparando-os ao mini-CEX, um por ser revisão da literatura, um por se tratar de revisão sistemática. Após a leitura integral dos 12 artigos elegíveis dessa segunda busca, dois foram incluídos no estudo final desta revisão sistemática e 10 foram excluídos por não se enquadrarem aos critérios de elegibilidade citados na metodologia, ou por não apontarem a validade do uso do mini-CEX na educação médica durante a graduação. A Figura 1 representa esse processo de seleção de artigos.

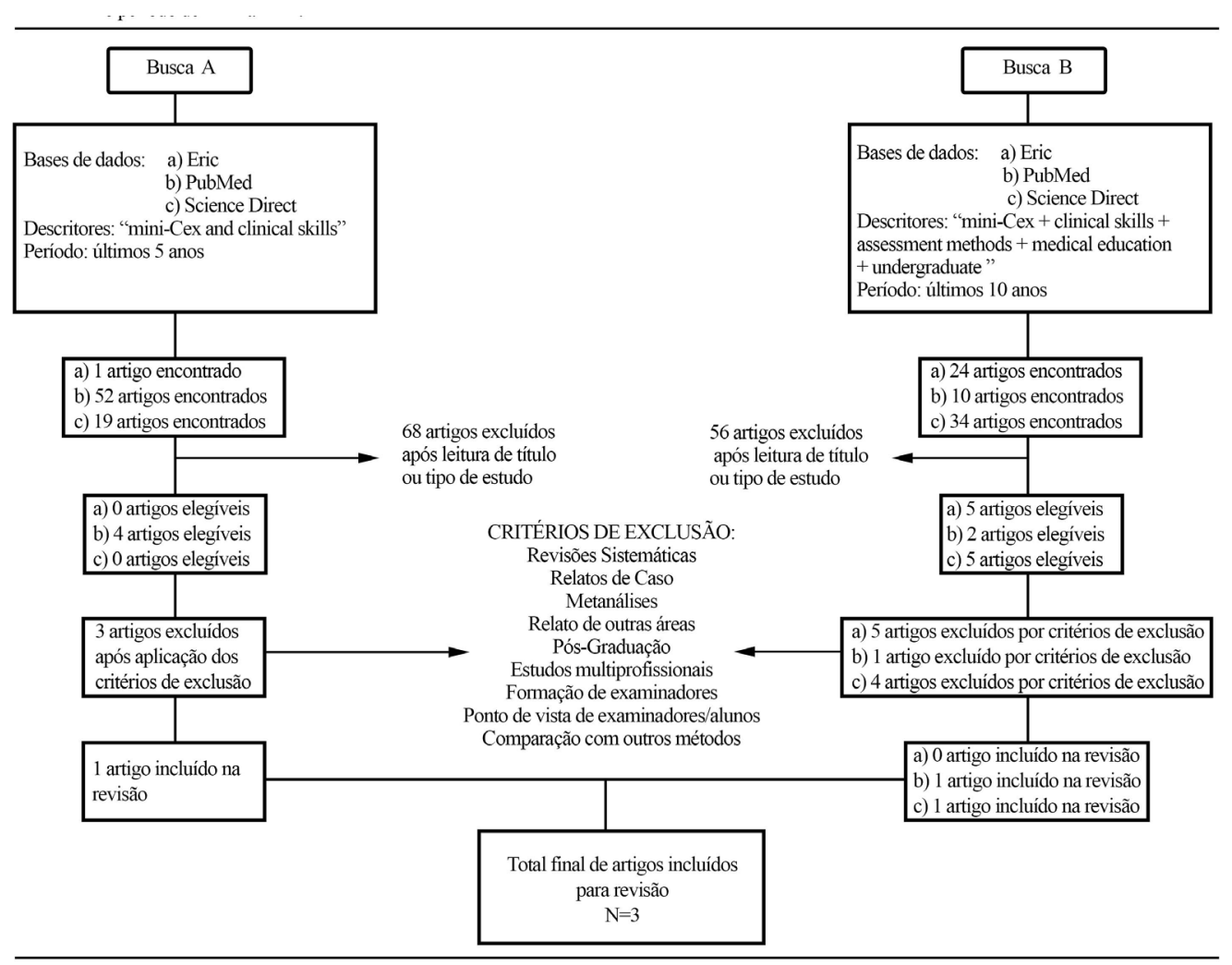

Figura 1. Fluxograma de pesquisa e seleção dos artigos para revisão sistemática sobre o uso do mini-CEX na avaliação de habilidades médicas na graduação, no período de 2006 a 2016

O estudo de Torre et al..$^{5}$ avaliaram a viabilidade das habilidades clínicas com uso do mini-CEX com 177 estudantes de medicina no terceiro ano do curso. Para tanto, cada acadêmico realizou dois mini-CEX em dois meses, totalizando 354 consultas analisadas no geral. Como conclusão, o estudo afirma que o uso do mini-CEX é um método viável para avaliação de habilidades clínicas tanto em ambiente hospitalar, quanto ambulatorial. Além disso, afirma que a observação direta da realização da consulta, a oportunidade do feedback ao fim de cada avaliação e a facilidade de agrupamento das competências do instrumento e suas análises são uma forma relevante de apoio para a avaliação formativa do aluno durante a formação médica.

Castro et al. ${ }^{6}$ avaliaram o mini-CEX com 926 estudantes de medicina, cada um realizando 4 avaliações com avaliadores diferentes. $\mathrm{O}$ estudo concluiu que dentre as sete competências avaliadas pelo instrumento, a anamnese, o exame físico, e a capacidade de comunicação são superiores a outros métodos de avaliação de competências objetiva e estruturada. Isso porque essa avaliação é feita em ambiente real com paciente não fictício, e favorece, sobremaneira, a relação médico/paciente. Além disso, o uso do feedback ao final da avaliação permite melhorar a qualidade das competências dos acadêmicos de medicina, favorecendo, assim, a melhor formação.

Quanto ao terceiro e último estudo avaliado, cuja autoria é de Hill et al. ${ }^{7}, 326$ estudantes foram avaliados em três ocasiões - e com avaliadores diferentes - quanto a anamnese, exame físico, raciocínio clínico e plano de manejo em áreas específicas da medicina, e satisfação de docentes e discentes. Como resultado, o exame físico obteve 
a menor resposta nas avaliações em pediatria e ginecologia com uso do mini-CEX, porém os comportamentos pessoal e profissional obtiveram as maiores pontuações gerais. Outro fator importante é que os acadêmicos e os professores avaliaram o mini-CEX como mais eficaz que outros métodos de avaliação de habilidades. Por fim, o estudo conclui que o uso de mini-CEX tem boa utilidade para avaliar aspectos do encontro clínico na graduação. Os três estudos concordaram que o mini-CEX na graduação, como método de avaliação de habilidades em consulta médica, mostrou-se relevante em relação a outros métodos, devido a sua característica bimodal de ser tanto avaliativo, quanto formativo. A Tabela 1 reflete a síntese do conteúdo dos três artigos selecionados.

Tabela 1. Especificidades dos estudos sobre a relevância da aplicação do mini-CEX na avaliação das habilidades clínicas em acadêmicos de medicina segundo autor, ano, local, duração do estudo, critérios avaliados, resultados e conclusões

\begin{tabular}{|c|c|c|c|c|}
\hline $\begin{array}{l}\text { Autor, Ano, } \\
\text { Referência }\end{array}$ & Local & $\begin{array}{l}\text { Duração do } \\
\text { Estudo }\end{array}$ & Critérios Avaliados & Resultados/Conclusões \\
\hline $\begin{array}{l}\text { Torre et al. }{ }^{5} \\
2007\end{array}$ & $\begin{array}{l}\text { Medical College of } \\
\text { Wisconsin (EUA) }\end{array}$ & $\begin{array}{l}\text { jul.2004 a abr. } \\
2005\end{array}$ & $\begin{array}{l}\text { Anamnese, exame físico, } \\
\text { profissionalismo, juízo } \\
\text { clínico, organização e plano } \\
\text { de manejo. }\end{array}$ & $\begin{array}{l}\text { Mini-CEX é viável e bem } \\
\text { avaliado por estudantes e } \\
\text { examinadores para supervisão e } \\
\text { aperfeiçoamento de habilidades } \\
\text { clínicas. }\end{array}$ \\
\hline $\begin{array}{l}\text { Castro et al. } \\
2015\end{array}$ & $\begin{array}{l}\text { International Conference } \\
\text { on University Teaching } \\
\text { and Innovation/ Facultad } \\
\text { de Medicina de la } \\
\text { Universitat Rovira i Virgili } \\
\text { (Tarragona - Espanha) }\end{array}$ & $\begin{array}{l}2002-2006 \\
2007-2013\end{array}$ & $\begin{array}{l}\text { Anamnese, exame físico, } \\
\text { comunicação, juízo clínico, } \\
\text { organização. }\end{array}$ & $\begin{array}{l}\text { Mini-CEX melhora habilidades } \\
\text { e competências clínicas e } \\
\text { comunicativas avaliadas na } \\
\text { graduação, favorecendo melhor } \\
\text { relação médico/paciente. }\end{array}$ \\
\hline $\begin{array}{l}\text { Hill et al. }{ }^{7} \\
2009\end{array}$ & $\begin{array}{l}\text { Southampton University } \\
\text { (Inglaterra) }\end{array}$ & $\begin{array}{l}\text { jul. } 2004 \text { a maio } \\
2005\end{array}$ & $\begin{array}{l}\text { Anamnese, exame físico, } \\
\text { juízo clínico, plano de } \\
\text { manejo. }\end{array}$ & $\begin{array}{l}\text { Mini-CEX é válido e confiável } \\
\text { na graduação. }\end{array}$ \\
\hline
\end{tabular}

\section{DISCUSSÃO}

Os estudos avaliados apresentaram metodologias diferentes, com aplicação de número variável de mini-CEX por estudante: Castro et al. ${ }^{6}$ realizaram quatro avaliações, Hill et al. ${ }^{7}$ realizaram três e Torre et al..$^{5}$ um total de duas, podendo essa variação influenciar na interpretação e nos resultados. Além disso, apenas um dos estudos especifica o ano cursado pelos estudantes avaliados, representando um viés de seleção ${ }^{5}$. Por outro lado, houve treinamento prévio dos avaliadores para aplicação do mini-CEX em cada estudo, contribuindo para redução do viés de informação, aumentando a confiabilidade dos resultados expostos ${ }^{5,6,7}$.

Cada trabalho apresentou limitações e dificuldades próprias: o estudo de Hill et al. ${ }^{7}$ demonstraram algumas variáveis externas de confusão, como rigidez do examinador, complexidade do caso, sexo do paciente e foco do caso. Torre et al. ${ }^{5}$ apresentaram limitações de recursos, falta de tempo, avaliação de estudantes de apenas uma instituição e o fato da aplicação do mini-CEX não ter sido realizada na presença do investigador do estudo para poder confirmar o conteúdo de cada exame clínico e validar o tipo e a qualidade do feedback recebido pelos alunos. E em Castro et al. $^{6}$ os quatro mini-CEX aplicados por aluno foram avaliados por tutores diferentes no estudo.

A presença do professor durante uma consulta médica proporciona maior segurança ao estudante de modo que se o acadêmico estiver com alguma dificuldade em conduzir a consulta o professor orienta-o como deve proceder através da avaliação do aluno pelo feedback. Isso corrobora com Megale et al. ${ }^{3}$ os quais afirmam ser indispensável que o professor observe diretamente as habilidades dos estudantes em ambiente real, pois só assim poderá afirmar que o aluno atingiu o nível de competência necessário ao seu aprendizado ${ }^{3-7}$. Megale et al. ${ }^{3}$ relataram ainda que o conhecimento teórico é a base para um diferencial na prática clínica, entretanto, somente a teoria sem a prática dificulta o manejo em situações de ação real. Através do mini-CEX, o estudante aprende a tomar decisões em situações de incerteza, a lidar com divergências, casos complexos e singulares, além de ter a segurança de estar sendo observado e tutelado pelo professor/avaliador que pode intervir caso haja necessidade durante a consulta ${ }^{3}$.

Dessa forma, percebe-se que o mini-CEX tem boa utilidade para avaliar as habilidades clínicas do estudante de graduação médica durante a consulta em um cenário de consulta real, sem pacientes simulados. Assim, o mini-CEX representa um método de avaliação que, além de sua fácil, prática e confiável aplicação, pode ser utilizado com caráter somativo e também formativo, mediante fornecimento de feedback ao final do exercício. No entanto, novos estudos mais robustos com estudantes de graduação médica e o uso do mini-CEX são necessários para detalhar seus pontos positivos e negativos, sobretudo quando comparado a outros métodos que avaliam o desenvolvimento de habilidades em estudantes de medicina. 
Agradecimentos: A Thiago Savona pela ajuda na elaboração das imagens.

Participação dos Autores: Diniz Neto AP, Lima CFT, Carvalho KRP e Santos RMP: idealização do desenho do estudo, pesquisa e revisão bibliográfica, redação e revisão. Lima RS, Fernandes RAML e Sales ACV: orientação, idealização do desenho do estudo, revisão e correção.

\section{REFERÊNCIAS}

1. Fornells-Vallés JM. El ABC del mini-CEX. Educ Med. 2009;12(2):83-9. doi: 10.4321/s1575-18132009000300004.

2. Baños JE, Gomar-Sancho C, Guardiola E, Palés-Argullós J. La utilización del Mini Clinical Evaluation Exercise (mini-CEX) en estudiantes de medicina. Rev Fund Educ Med. 2015;18(16):417-26. doi: 10.4321/S201498322015000700010 .

3. Megale L, Gontijo ED, Motta JAC. Avaliação de competência clínica em estudantes de medicina pelo Miniexercício Clínico Avaliativo (Miniex). Rev Bras Educ Med. 2009;33(2):166-75. doi: 10.1590/S0100-55022009000200002.

4. Higgins JPT, Green S. Cochrane handbook for systematic reviews of interventions. Version 5.1.0 [updated March 2011]. The Cochrane Collaboration, 2011. Available from: http:// www.cochrane-handbook.org. doi: 10.1002/jrsm.38.
5. Torre DM, Elnicki DM, Sebastián JL, Holmboe ES. Feasibility, reliability and user satisfaction with a PDA-based mini-CEX to evaluate the clinical skills of third-year medical students. Teach Learn Med. 2007;19(3):271-7. doi: 10.1080/ 10401330701366622.

6. Castro A, Descarrega R, Parra S, Odeja R, Roma J, Vidal F, et al. Las competencias profesionales de los titulados en medicina mejoran con la introducción de un programa de simulación con pacientes estandarizados y MINIC-CEX. Procedia - Social Behav Sci. 2015;196:25-9. doi: 10.1016/j. sbspro.2015.07.005.

7. Hill F, Kendall K, Galbraith K, Crossley J. Implementing the undergraduate mini-CEX: a tailored approach at Southampton University. Med Educ. 2009;43(4):326-34. doi: $10.1111 /$ j.1365-2923.2008.03275.x.

Recebido: 22.04.18

Aceito: 26.11 .19 